Gesnerus 55 (1998) 331-336

\title{
Contents of Vol. 55 (1998)
}

\section{Original Articles}

Dasen, Véronique: Les naissances multiples dans les textes médicaux antiques [Multi-

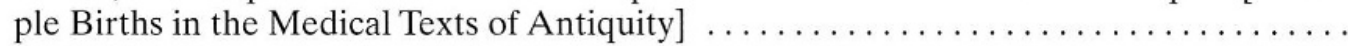

Diosi, Peter: Das Enträtseln der Zelleinschlüsse. Ein Beitrag zur Geschichte der Virologie [Interpreting Cellular Inclusions. A Contribution to the History of Virology] . . . . .

Ebrahimnejad, Hormoz: La médecine d'observation en Iran du XIX ${ }^{\mathrm{e}}$ siècle [Observa-

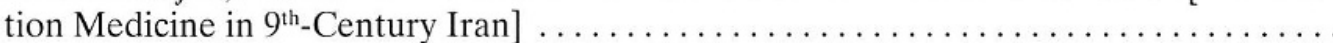

Gross, Dominik: Der Beitrag Gottlieb Burckhardts (1836-1907) zur Psychochirurgie in medizinhistorischer und ethischer Sicht [Gottlieb Burckhardt's (1836-1907) Contribution to Psychosurgery. Medicohistorical and Ethical Aspects] ...................

Jagella, Caroline: Eugen Bleulers Warnung vor dem «autistisch-undisziplinierten Denken in der Medizin» als Beitrag zur Erkenntniskritik ärztlicher Forschung. Ein Zürcher Konzept über «ratio et experientia» im ärztlichen Denken [Eugen Bleuler's Warning of "Autistic and Undisciplined Thinking in Medicine"] ........................

Jeanmonod, Gilles: Mutation du concept de dégénérescence en Suisse romande 1870-1920 [Shifting Concepts of Degeneration in French-Speaking Switzerland

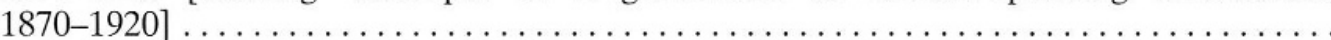

Mannetstätter, Antje; Friedrich, Christoph: Die Zürcher Arzt-Apotheker-Familie Lavater und Johann Wolfgang von Goethe [The Lavater of Zurich, a Family of Physicians and Pharmacists. Their Connection with Goethe] $\ldots \ldots \ldots \ldots \ldots \ldots \ldots \ldots \ldots . . . \ldots \ldots$

Müller, Christian: Die Drehmaschinen in der Geschichte der Psychiatrie [The Rotatory

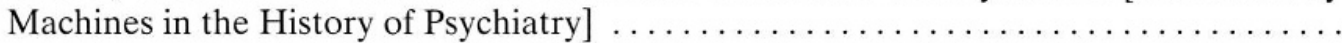

Müller, Jürgen: Hexensalben und Liebestränke. Ein Beitrag zur Kulturgeschichte der Nachtschattengewächse [Witches' Ointments and Love-potions. A Contribution to the Cultural History of Nightshades $] \ldots \ldots \ldots \ldots \ldots \ldots \ldots \ldots \ldots \ldots \ldots \ldots \ldots \ldots$.

Toplak, Cirila; Zupanič-Slavec, Zvonka: Water, Air and Light - Arnold Rikli

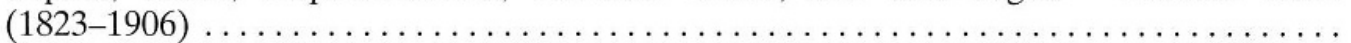

Walser, Hans H.: Zum 10. Todestag von Erwin H. Ackerknecht (1906-1988) [Remembering E. H. Ackerknecht on the $10^{\text {th }}$ Anniversary of his Death] 


\section{Editorial}

The Editorial Board Completing its Fifth Year $\ldots \ldots \ldots \ldots \ldots \ldots \ldots \ldots \ldots \ldots \ldots$

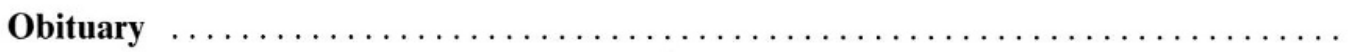

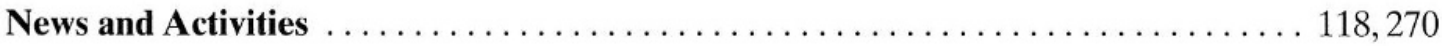

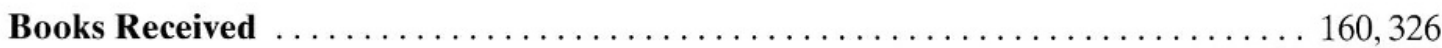

\section{Book Reviews}

Appell, Rainer G. (Hrsg.): Homöopathie zwischen Heilkunde und Heilkunst

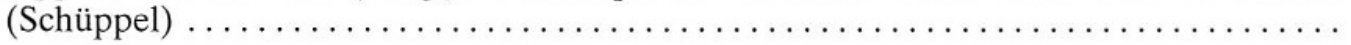

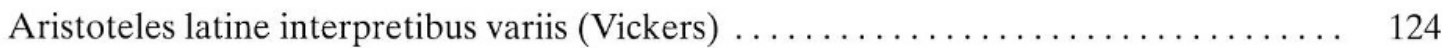

Bäumer, Änne: Bibliography of the history of biology.-Bibliographie zur Geschichte der

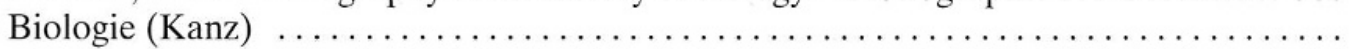

Beretta, Marco: The starry messenger and the Polar star (Bröer) ….......... 276

Berger, Georg: Die Beratenden Psychiater des deutschen Heeres 1939 bis 1945

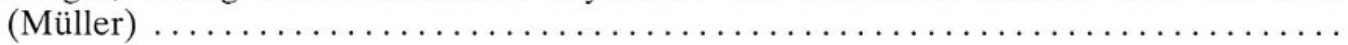

Bernoulli: Die gesammelten Werke der Mathematiker und Physiker der Familie Bernoulli. Die Werke von Daniel Bernoulli, Bd. 1 (Gantenbein) ....................

Bien, Christian F.: Erklärungen zur Entstehung von Missbildungen im physiologischen

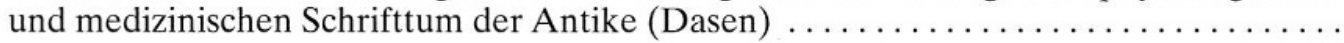

Blanc, Jean Daniel: Wachstum und Wachstumsbewältigung im Kanton Basel-Landschaft

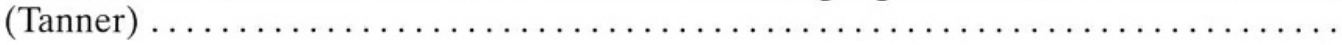

Bosson, Alain: Histoire des médecins fribourgeois (1850-1900). Des premières anesthé-

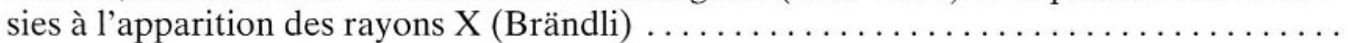

Brunner, Christoph H.: «Der Saft von Bittersüss». Medizin in Glarus um 1800 (Stein-

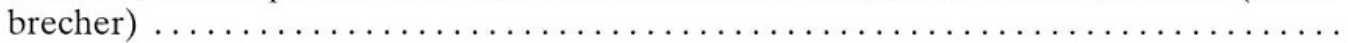

Bueltzingsloewen, Isabelle von: Machines à instruire, machines à guérir. Les hôpitaux universitaires et la médicalisation de la société allemande (1730-1850) (Loetz) ........

Capuano, Fabrizia; Manzini, Paola (eds.): La «Mal-Aria» di Lazzaro. Spallanzani e la

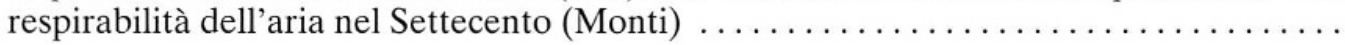

Degen-Zimmermann, Dorothee: Mich hat niemand gefragt. Die Lebensgeschichte

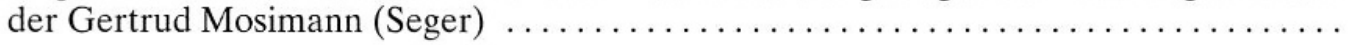

Dehmel, Gisela: Die Arzneimittel in der Physikotheologie (Walser) . . . . . . . . . 282

Dilg, Peter; Rudolph, Hartmut: Neue Beiträge zur Paracelsus-Forschung (Gantenbein) . 
Dilg, Peter; Rudolph, Hartmut (Hrsg.): Resultate und Desiderate der Paracelsus-

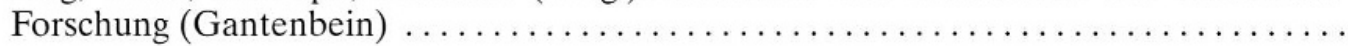

Dührssen, Annemarie: Ein Jahrhundert Psychoanalytische Bewegung in Deutschland

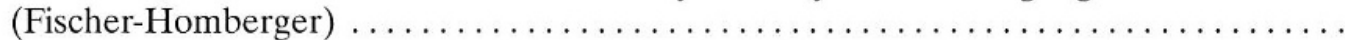

Duffin, Jacalyn: To see with a better eye. A life of R. T. H. Laennec (Mayer) . . . . . . . 283

Eckart, Wolfgang U.: Geschichte der Medizin (Seger) $\ldots \ldots \ldots \ldots \ldots \ldots \ldots \ldots \ldots \ldots$

The emergence of modern physics (Kleinert) $\ldots \ldots \ldots \ldots \ldots \ldots \ldots \ldots \ldots \ldots \ldots \ldots .284$

Enz, Charles P.; Glaus, Beat; Oberkofler, Gerhard (Hrsg.): Wolfgang Pauli und sein Wir-

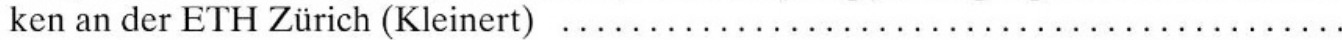

Erinnern und Behalten. Wege zur Erforschung des menschlichen Gedächtnisses

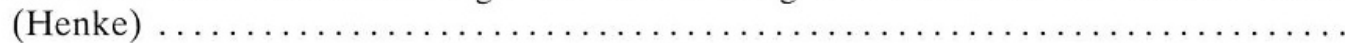

Estes, J. Worth: Naval surgeon. Life and death at sea in the age of sail (Bickel) .......

Fahrenbach, Sabine; Wiedemann, Peter: Augenheilkunde in Leipzig. Von der «Heilanstalt für arme Augenkranke» zur modernen Universitätsklinik (Koelbing) . . . . . . . . .

Farber, Stephen; Green, Marc: Hollywood on the Couch. A candid look at the overheated love affair between psychiatrists and moviemakers (Fischer-Homberger) ......

Fehlmann, Sabine Irène: Deutsche Apotheker in der Schweiz (Simon)

Ferrari, Giovanna: L'esperienza del Passato. Alessandro Benedetti, filologo e medico

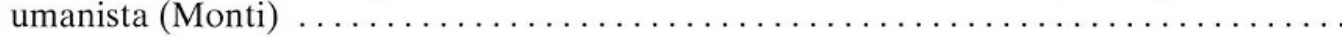

Finzsch, Norbert; Jütte, Robert (eds.): Institutions of confinement. Hospitals, asylums, and prisons in Western Europe and North America 1500-1950 (Steinbrecher) . . . . . . .

Fischer, Roman: Die Trepanations-Metapher. Über den literarischen Umgang mit einem

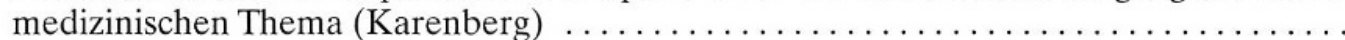

Grmek, Mirko D.: Il calderone di Medea. La sperimentazione sul vivente nell' An-

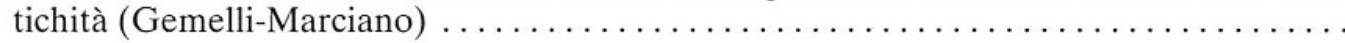

Gros, Frédéric: Création et folie. Une histoire du jugement psychiatrique (Courbet) ...

Hagelin, Ove (compiler): Old and rare books on Materia Medica in the Library of the Swedish Pharmaceutical Society [Apotekarsocieteten] (Seger) $\ldots \ldots \ldots \ldots \ldots \ldots$

Hagner, Michael: Homo cerebralis. Der Wandel vom Seelenorgan zum Gehirn

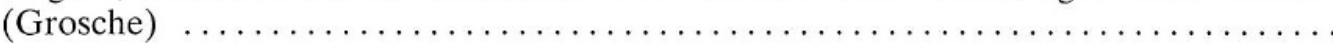

Hernschier, Wolfgang: Ich wollte, ich wäre ein guter Schuhflicker ... Das unglückliche Leben des bayerischen Astronomen Johann Nepomuck Fischer (1749-1805) (Ganten-

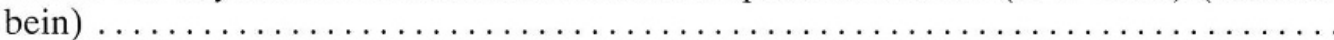

Hess, Volker (Hrsg.): Normierung der Gesundheit. Messende Verfahren der Medizin als

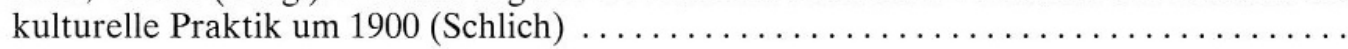

Hiller, Helmut: Paracelsus-Lexikon (Gantenbein) $\ldots \ldots \ldots \ldots \ldots \ldots \ldots \ldots \ldots \ldots \ldots$ 
Huisman, Frank; Santing, Catrien (eds.): Medische geschiedenis in regionaal perspectief:

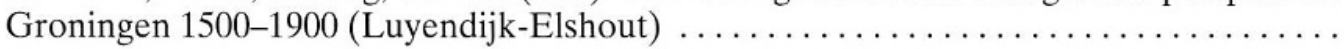

Juch, Alice: De medisch specialisten in de Nederlandse gezondheidszorg (Luyendijk-

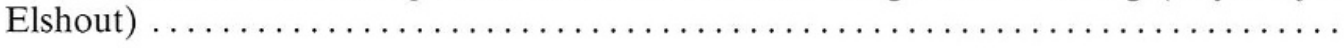

Jütte, Robert (Hrsg.): Paracelsus heute - im Lichte der Natur (Gantenbein)

Kanz, Kai Torsten: Nationalismus und internationale Zusammenarbeit in den Natur-

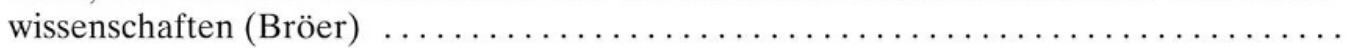

Klassische tibetische Medizin (Gantenbein) $\ldots \ldots \ldots \ldots \ldots \ldots \ldots \ldots \ldots \ldots \ldots \ldots \ldots \ldots \ldots$

Klee, Ernst: Auschwitz, die NS-Medizin und ihre Opfer (Grosche) $\ldots \ldots \ldots \ldots \ldots \ldots 297$

Köchy, Kristian: Ganzheit und Wissenschaft. Das historische Fallbeispiel der roman-

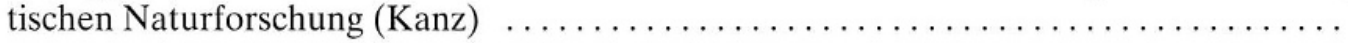

Krätz, Otto: Alexander von Humboldt. Wissenschaftler - Weltbürger - Revolutionär

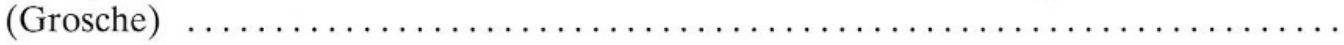

La Berge, Ann; Feingold, Mordechai (eds.): French medical culture in the nineteenth

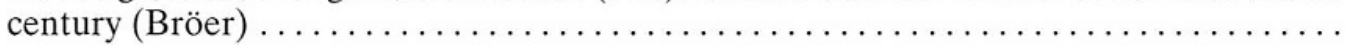

Lamens-van Malenstein, M. M.: Oefening en bespiegeling. Het verloskunde onderwjis von M. S. du Pui (1754-1834) te Leiden (Luyendijk-Elshout) .................

Lanz, Almut: Arzneimittel in der Therapie Friedrich Hoffmanns (1660-1742)

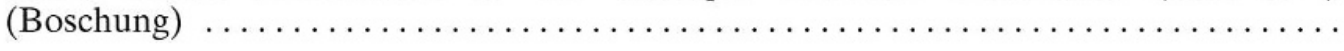

Lavoisier, Antoine Laurent: Correspondance, vol. VI, 1789-1791 (Mayer) ...........

Lehrach, Dirk: Wiederaufbau und Kernenergie. Zur Haltung deutscher Emigranten in

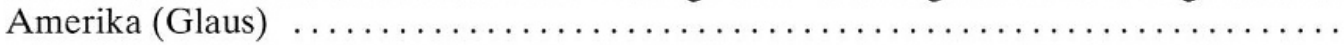

Leuenberger, Martin: Frei und gleich ... und fremd (König) . . . . . . . . . . . . . . 141

Leven, Karl-Heinz: Die Geschichte der Infektionskrankheiten (Ritzmann) . . . . . . 142

Lieburg, M. J. van: De geschiedenis van de kindergeneeskunde in Nederland (Huizink)

Luginbühl-Weber, Gisela: Johann Kaspar Lavater - Charles Bonnet - Jacob Bennelle: Briefe 1768-1790 (Boschung) . . . . . . . . . . . . . . . . . . . . . . .

Meier, Pirmin: Ich Bruder Klaus von Flüe (Koelbing) $\ldots \ldots \ldots \ldots \ldots \ldots \ldots \ldots$

Meinel, Christoph; Voswinckel, Peter (Hrsg.): Medizin, Naturwissenschaft, Technik

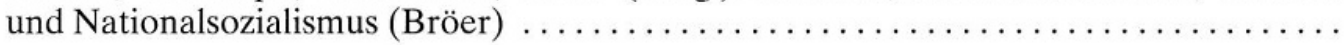

Müller-Landgraf, Ingrid; Ledermann, François: Medizin und Pharmazie in Bern

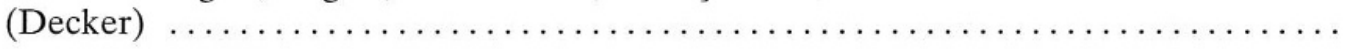

Murken, Axel Hinrich (Hrsg.): 150 Jahre St. Hedwig-Krankenhaus in Berlin, 1846-1996

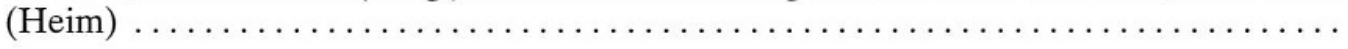

Neumann, Herbert A.: Vom Ascaris zum Tumor: Leben und Werk des Biologen Theodor

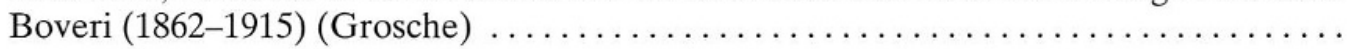




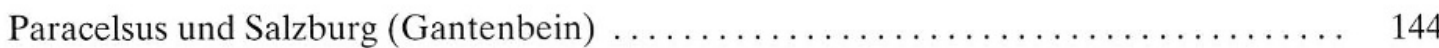

Paulus, Julian: Paracelsus-Bibliographie 1961-1996 (Gantenbein) . ............. 145

Per una storia critica della scienza $($ Bergdolt $) \ldots \ldots \ldots \ldots \ldots \ldots \ldots \ldots \ldots \ldots \ldots \ldots \ldots \ldots$

Pfeiffer, Jürgen: Hirnforschung im Zwielicht: Beispiele verführbarer Wissenschaft aus

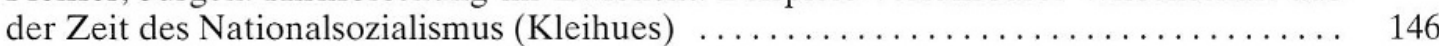

Premuda, Loris: Da Fracastoro al Novecento. Mezzo millennio di medicina tra Padova,

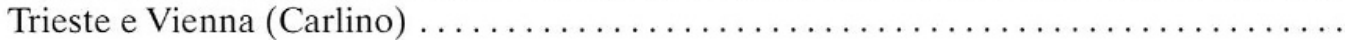

Priesner, Claus: Bayerisches Messing. Franz Matthias Ellmayrs «Mössing-Werkh AO.

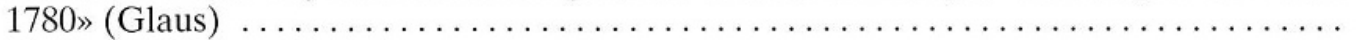

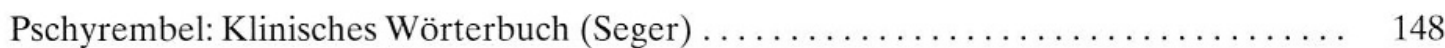

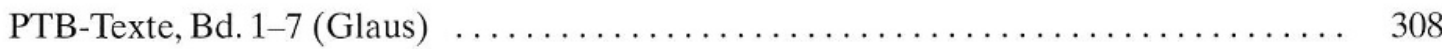

Rattansi, Piyo; Clericuzio, Antonio (eds.): Alchemy and chemistry in the $16^{\text {th }}$ and $17^{\text {th }}$

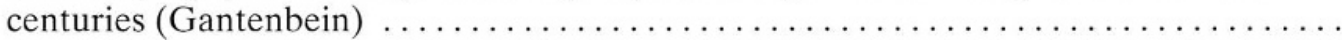

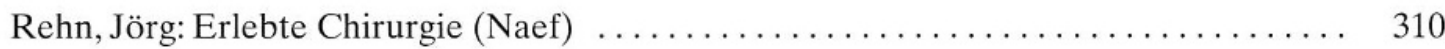

Rippmann, Dorothee; Simon-Muscheid, Katharina; Simon, Christian: Arbeit - Liebe -

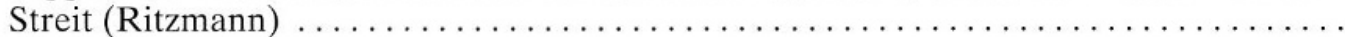

Röell, D. R.: De wereld van instinct. Niko Tinbergen en het ontstaan van de ethologie in Nederland (1920-1950) (Isler) . . . . . . . . . . . . . . . . . . . . . . . .

Rueb, Franz: Hexenflug und Teufelsritt (Utz Tremp) $\ldots \ldots \ldots \ldots \ldots \ldots \ldots \ldots \ldots \ldots$

Ryter, Annamarie: Als Weibsbild bevogtet. Zum Alltag von Frauen im 19. Jahrhundert

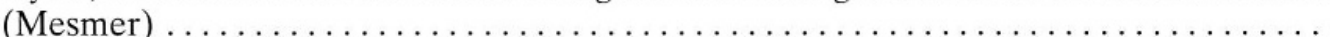

Sachs, Michael: Historisches Ärztelexikon für Schlesien, Bd. 1: A-C (Kanz) . . . . . . . . 311

Schett, Alfred: The ophthalmoscope - Der Augenspiegel (Koelbing) . . . . . . . . . 312

Schliack, Hans; Hippius, Hanns (Hrsg.): Nervenärzte (Müller) … . . . . . . . . . 313

Schnalke, Thomas: Medizin im Brief. Der städtische Arzt des 18. Jahrhunderts im Spiegel seiner Korrespondenz (Steinke) . . . . . . . . . . . . . . . . . . . . . . . . .

Seidel, Hans-Christoph: Eine neue «Kultur des Gebärens». Die Medikalisierung von Geburt im 18. und 19. Jahrhundert in Deutschland (Gamper) .................

Seidensticker, Peter: die seltzamen namen all (Keil) . . . . . . . . . . . . . . . . . 152

Sievert, Lars Endrik: Naturheilkunde und Medizinethik im Nationalsozialismus

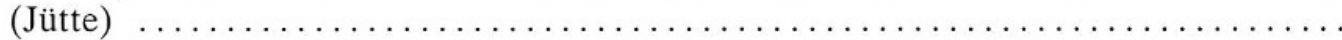

Spallanzani, Lazzaro: Edizione nazionale delle opere di Lazzaro Spallanzani, parte 4: Opere edite direttamente dall'autore, vol. I (1760-1768); vol. II (1768-1773) (Boschung)

Spitzer, Gabriele: ... und die Spree führt Gold (Gantenbein) $\ldots \ldots \ldots \ldots \ldots \ldots \ldots \ldots$ 
Steeno, Omer: Johannes-Remigius Jacquelart uit Nijvel (1721-1809) (Luyendijk-

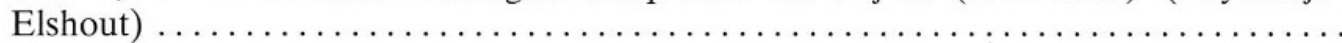

Stefenelli, Norbert (Hrsg.): Körper ohne Leben. Begegnung und Umgang mit Toten

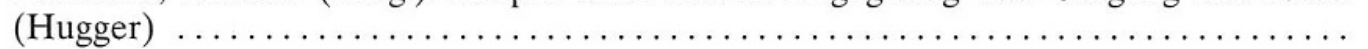

Stensen, Niels: Chaos. Niels Stensen's Chaos-manuscript - Copenhagen, 1659 (Kanz) . .

Stolberg, Michael: Die Cholera im Grossherzogtum Toskana. Ängste, Deutungen und Reaktionen im Angesicht einer tödlichen Seuche (Gamper) ...................

Strauss, Elisabeth (Hrsg.): Dilettanten und Wissenschaft (Bröer) . . . . . . . . . . . . 319

Sutter, Pascale: «Arme Siechen» (Rickenbacher) $\ldots \ldots \ldots \ldots \ldots \ldots \ldots \ldots \ldots \ldots \ldots$

Take time by the forelock: the letters of Anthony Fothergill to James Woodforde,

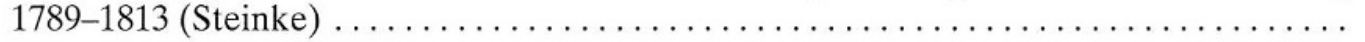

Tenon, Jacques: Memoirs on Paris Hospitals (Louis-Courvoisier) . . . . . . . . . . . . . 321

Trus, Armin: «Ö vom Leid erlösen». Zur Geschichte der nationalsozialistischen «Eutha-

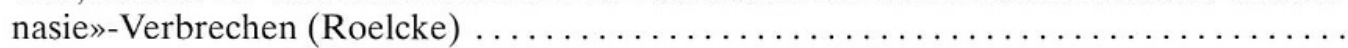

Ullmann, Dirk: Quelleninventar Max Planck (Glaus) $\ldots \ldots \ldots \ldots \ldots \ldots \ldots \ldots \ldots$

Wahl, Gunter; Schmitt, Wolfram (Hrsg.): Heilen - Verwahren - Vernichten (Müller) ...

Widmann, Martin; Mörgeli, Christoph: Bader und Wundarzt. Medizinisches Handwerk

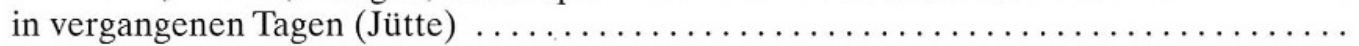

Winkle, Stefan: Geisseln der Menschheit (Leven) $\ldots \ldots \ldots \ldots \ldots \ldots \ldots \ldots \ldots \ldots \ldots \ldots$

Woodward, John; Jütte, Robert (eds.): Coping with sickness. Perspectives on health care,

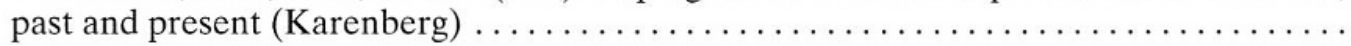

Wunderlich, Uli: Ubique Holbein: Drei Totentanzwerke aus drei Jahrhunderten

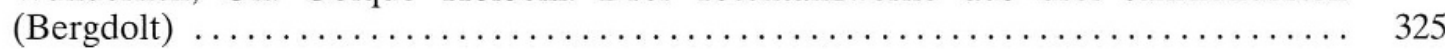

Zimmermann, Volker (Hrsg.): Paracelsus. Das Werk - die Rezeption (Gantenbein) … 158 